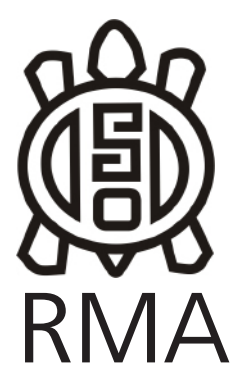

Antropología Biológica

\title{
Desplazamiento étnico en la Región Bonaerense. Empleo de marcadores genéticos en la detección del nivel de heterogeneidad genética en la población actual
}

\author{
María Cecilia Bobillo* y Daniel Corach* \\ *Servicio de Huellas Digitales Genéticas y Cátedra de Genética \\ y Biología Molecular, Facultad de Farmacia y Bioquímica, \\ Universidad de Buenos Aires. E-mail: cebobillo@ffyb.uba.ar
}

\begin{abstract}
Resumen
Actualmente en la provincia de Buenos Aires, con una población de más de 15 millones de habitantes, los pueblos aborígenes representan el 0,36\%. Desde mediados de los '40, tanto los aborígenes y sus descendientes, como los inmigrantes europeos y latinoamericanos instalados en el interior del país fueron migrando tanto a la ciudad de Buenos Aires como a su conurbano, fenómeno fomentado por la actividad industrial y agrícola-ganadera.

Con el objeto de evaluar el aporte de los distintos grupos étnicos a la composición genética de la población actual de la provincia de Buenos Aires (centrándonos en CABA y conurbarno), llevamos a cabo el análisis de marcadores genéticos de herencia uni y biparental (ADN mitocondrial: región de control y SNPs de región codificante, Cromosoma Y: SNPS y SNPs localizados en cromosomas autosómicos). Los resultados obtenidos permiten interpretar los procesos migratorios y de mestizaje que co-participaron en la constitución actual de la provincia, cuya población representa el 52\% de la población total del país. De estos resultados surge un claro efecto de desplazamiento étnico ya que después de 20 generaciones del primer contacto no es posible detectar individuos que mantengan la estructura genética de las poblaciones originarias de América.
\end{abstract}

Palabras clave: Desplazamiento étnico; marcadores genéticos; región Bonaerense.

Ethnic Displacement in Buenos Aires Region. Detection of Genetic Heterogeneity level in the current Population using genetic markers

\begin{abstract}
In the province of Buenos Aires, with a population of over 15 million inhabitants, aboriginal people currently account for $0.36 \%$. Since the mid-40s, aborigines and their descendants, as well as European and Latin American immigrants, [comma] settled in the major urban areas, such as Buenos Aires city and its suburbs, fueled by industrial, agricultural and livestock activities.

In order to evaluate the contribution of the various ethnic groups to the genetic make-up of the current population of Buenos Aires province, we analyzed uni- and biparental inheritance markers (mitochondrial DNA: control region and coding region SNPS, Y chromosome: SNPs and SNPs located on autosomal chromosomes). The results allow interpreting the processes of migration and mixing of cultures that co-participated in the present genetic constitution of the province that accounts for $52 \%$ of the total population of Argentina. Findings suggest a clear effect of ethnic displacement considering that at present, 20 generations after the first contact; it is not possible to detect individuals maintaining the genetic make-up of the pre-Hispanic populations of America
\end{abstract}

Keywords: Ethnic displacement; genetic markers; Buenos Aires region.

Se estima que la población aborigen al momento del primer contacto de los conquistadores europeos, en 1516, ascendía a 400.000 habitantes distribuidos en el actual territorio de Argentina. Los Tehuleches y Querandíes habrían representado los grupos mayoritarios en la región bonaerenese (Martínez Sarasola 2011). La conquista tuvo un drástico impacto sobre la demografía de las poblaciones aborígenes del continente americano, resultando en una reducción de las poblaciones locales a través de enfermedades, guerras y trabajos extenuantes, que particularmente afectó a la población masculina; en tanto que las mujeres fueron sojuzgadas y proporcionaron la matriz que generó la población mestiza.

Antes de 1810, tanto la población de europeos nativos como de esclavos africanos había aumentado paulatinamente en el actual territorio de Buenos Aires. (Mandrini 2005, 2008, Martínez Sarasola 2011). Desde el año 1534, y con mayor intensidad durante el breve periodo virreinal (1776-1810), llegaron al puerto de 
Buenos Aires africanos occidentales traídos como esclavos, estimando el ingreso de unos 100.000 esclavos. (Rowley 2005).

Entre los años 1810 y 1887 se observó una reducción considerable de la población africana y sus descendientes en el territorio de la actual Buenos Aires, pasando de representar del $25 \%$ al $1,8 \%$ de la población total. Esta disminución podría atribuirse a tres factores: a- la "Libertad de Vientres" de la Asamblea de 1813, b- los reclutamientos de la población masculina afrodescendiente para las guerras de la independencia (Andrews 1980) y c- la prohibición del trafico de esclavos consecuente con la abolición de la esclavitud en 1853, sin embargo estos motivos son actualmente discutidos.

Entre 1850 y 1930 Argentina recibió una inmigración masiva proveniente de Europa, principalmente de Italia y España. El notable aporte europeo se debió a una política inmigracionista convocante, claramente reflejada en el artículo 25 de la Constitución Nacional de 1853, y a la situación socio-económica de los países europeos. Durante este período el número de inmigrantes ascendió a 6.000.000. En el censo de 1914 el 30\% de la población argentina era extranjera, radicada preferentemente en las ciudades, pudiéndose registrar más del 49\% de extranjeros en la ciudad de Buenos Aires (La Ciudad en el Censo Nacional de 1914).

Durante el siglo veinte, además del aporte inmigratorio europeo, nuestro territorio recibió contribuciones provenientes de países limítrofes. El censo de 2010 (INDEC), registra una población total para la República Argentina de 40.117.096 habitantes. De este total el $4,5 \%$ son nacidos en el exterior y un total de 600.329 personas se reconocen pertenecientes y/o descendientes en primera generación de pueblos indígenas (Registro Nacional de Comunidades Indígenas - RE.NA.C.I -Encuesta Complementaria de Pueblos Indígenas 2004-2005).

Actualmente en la provincia de Buenos Aires, con una población de más de 15 millones de habitantes, los pueblos aborígenes representan el 0,36\% y se localizan mayoritariamente en las tres coronas del conurbano bonaerense (Avena et al. 2001). Desde mediados de los '40, tanto los aborígenes y sus descendientes, como los inmigrantes europeos y latinoamericanos instalados en el interior del país fueron migrando tanto a la ciudad de Buenos Aires como a su conurbano. Este cambio demográfico fue el resultado de políticas que fomentaron la actividad industrial y agrícola-ganadera.

El empleo de marcadores genéticos ubicados en el cromosoma Y, en el ADN mitocondrial, y aquellos presentes en los cromosomas autosómicos permiten evaluar cuáles han sido los principales actores determinantes de las contribuciones étnicas a la población actual de la provincia de Buenos Aires. Los marcadores de herencia uniparental, incluyendo los de la Región de Control del ADN mitocondrial y la región no recombinantes del cromosoma Y (NRY), permiten inferir el origen étnico/geográfico del linaje al que pertenece un individuo, debido a sus características de transmisión (Cann et al. 1987, Vigilant et al. 1991, De Knijff 2000, Underhill y Kivisild 2007). En tanto que el análisis de sistemas recombinantes, como los ubicados en cromosomas autosómicos, permiten evaluar los procesos de mestizajes ocurridos a través del tiempo (Kersbergen et al. 2009, Corach et al. 2010)

Con el objeto de evaluar el aporte de los distintos grupos étnicos a la composición genética de la población actual de la provincia de Buenos Aires llevamos a cabo el análisis de marcadores genéticos de herencia uni y biparental. Los resultados obtenidos permiten interpretar los procesos migratorios y de mestizaje que co-participaron en la constitución actual de la provincia, cuya población representa el 52\% de la población total del país.

\section{Materiales y métodos}

\section{Individuos}

Se analizaron 154 muestras de sangre de individuos de sexo masculino, que al momento de la toma de muestra se domiciliaban tanto en CABA (44,3\%), como en provincia de Buenos Aires (47,9\%), principalmente en el conurbano. En 13 muestras no pudo constatarse la pertenencia a CABA o a provincia de Buenos Aires. En todos los casos las muestras corresponden a individuos que solicitaron, ya sea en forma particular o a través de la justicia, estudios de paternidad.

Todos los donantes de las muestras leyeron y firmaron un acta de consentimiento informado que cuenta con la aprobación del Comité de Ética de la Facultad de Farmacia y Bioquímica de la Universidad de Buenos Aires

\section{Muestras}

Las muestras de sangre se obtuvieron por punción digital. Se depositaron de 5 a 10 gotas de sangre sobre papel FTA, las cuales fueron secadas a temperatura ambiente y mantenidas en esta condición hasta el momento de su procesamiento.

\section{Extracción de ADN}

La preparación de las muestras de ADN fijado en el soporte FTA se realizó mediante el empleo del reactivo de extracción según indicaciones del fabricante (www. whatman.com/DNACollection.aspx).

\section{Marcadores genéticos}

\section{Herencia biparental}

Se analizaron polimorfismos de sustitución específicos 
de ancestro (SNPS-AIMs) localizados en cromosomas autosómicos. Se analizaron los 24 SNPs seleccionados y caracterizados por Lao et al. (2009) (códigos de Genbank: rs1876482, rs2179967, rs1048610, rs1371048, rs1478785, rs1369290, rs952718, rs1405467, rs1344870, rs1391681, rs1461227, rs1907702, rs2052760, rs714857, rs721352, rs722869, rs926774, rs1448484, rs1667751, rs1858465, rs1465648, rs16891982, rs1808089 y rs3843776) empleando la técnica de mini secuenciación SNaPshot ${ }^{\circledR}$ (Applied Biosystems, Foster City, USA). La electroforesis capilar se llevo a cabo en el secuenciador automático ABI3100-Avant (Applied Biosystems, Foster City, USA) utilizándose para el análisis de los resultados el programa GeneMapper ID 3.2 (Applied Biosystems, Foster City, USA).

\section{Herencia uniparental materna}

Se analizaron la totalidad de las muestras mediante la secuenciación de la región de control completa del ADN mitocondrial, desde la posición 16024 a la 576, se determinaron los SNPs $(12468,3547,6755,1888$, 7697, 2766, 8383, 8419, 9431, 5319, 7028, 2706, 9716, 14798, 4580, 12308 y 12372) ubicados en la región codificante, que permitieron definir subhaplogrupos en algunas de las muestras estudiadas.

Región de control: la amplificación se llevó a cabo en el ciclador térmico 9700 GeneAmp (Applied Biosystems, Foster (ity, Ca.USA) utilizando 0,5 uM de los primers L15971 y H576. La secuenciación se hizo utilizando el sistema BigDye Terminator v1.1, en dirección Forward y Reverse utilizando los primers: L15971, L15989, L16268, L15, L314, L361, H16, H159, H484 y H639 para cada muestra de acuerdo a Brandstätter et al. (2006)

Región codificante: la detección de SNPs se realizó utilizando la técnica de SNaPshot ${ }^{\circledR}$ usándose la estrategia empleada con los SNP-AIMs autosómicos.

\section{Herencia uniparental paterna}

La región no recombinante del cromosoma $Y$ se analizó mediante el uso de 24 SNPS (SRY 1532, M91, M168, M145, M174, 12f2, M96, M213, M201, M69, M52, M170, M172, M9, M20, M106, M214, Tat, M175, M45, MEH2, M207, M269 y M124) que fueron evaluados en todas las muestras, empleándose la técnica de SNaPshot ${ }^{\circledR}$ (Applied Biosystems, Foster City, USA). También se analizó el SNP M3-DYS199, cuya variante alélica T es característica de las poblaciones pre-hispánicas, utilizando la técnica de PCR en tiempo real seguida por desnaturalización de alta resolución (Zuccarelli et al. 2011)

\section{Análisis estadísticos}

\section{SNPS-AIMs autosómicos}

Se utilizó el programa estadístico STRUCTURE (Pritchard et al. 2000) para evaluar la composición étnica comparándose con poblaciones parentales del panel del
Human Genome Diversity Project, Centre d'Études des Polymorphismes Humaines -HGDP-GEPH- (158 muestras de origen europeo, 44 nativos de Sudamérica y 49 de África occidental sub-sahariana). La distancia genética entre poblaciones se calculó utilizando la matriz de distancia IBS y los resultados de distancia obtenidos fueron graficados mediante escalado multidimensional (MDS) usándose el programa SPSS 15.0 (SPSS for Windows, Rel. 15.0.1. 2006. SPSS Inc., Chicago, Illinois, USA).

\section{ADN mitocondrial}

Las secuencias se analizaron mediante el programa Sequencing Analysis (Versión 5.1 Applied Biosystem, $A B$, Foster City, CA, USA) y luego fueron ensambladas y comparadas con la secuencia de referencia revisada de Cambridge (rCRS), utilizando el programa Sequencher v4.9 (Gene Codes Corporation, USA).

La asignación de haplogrupos se realizó teniendo en cuenta la guía para la nomenclatura de Bandelt y Parson (2008) basándose en las mutaciones observadas en las regiones HVR I, II y III. Para el análisis de variación de secuencia (Arlequín 3.11) se tuvo en cuenta el número de sustituciones y transversiones, la presencia de inserciones y deleciones, y los distintos puntos heteroplásmicos. También se evaluó la presencia de haplotipos distintos, únicos y repetidos.

El programa Network 4.516 (Fluxus) se utilizó para graficar en forma de red la relación entre los distintos haplogrupos y subhaplogrupos con los nodos principales, basado en los pasos de mutación observados.

\section{Resultados}

\section{SNPs-AIMs autosómicos}

Los resultados de distancia IBS se graficaron mediante escalado multidimensional (Figura 1), observándose distancias similares entre las tres poblaciones parentales, indicando que el conjunto de AIMs empleados poseen un poder discriminante adecuado para diferenciar las poblaciones ancestrales seleccionadas. La mayoría de las muestras de Buenos Aires se agruparon junto con o muy cerca de la población europea, observándose algunos individuos entre europeos y nativos americanos indicando mezcla génica entre estos dos grupos.

Para evaluar la composición étnica a nivel individual se utilizó el programa STRUCTURE. Mediante este análisis pudo observarse un patrón similar donde la mayoría de las muestras revelaron un ancestro europeo mayoritario, si bien fue observada una fracción considerable de ancestro amerindio en muchas de las muestras estudiadas (Figura 2).

La proporción de cada componente se resume en la 


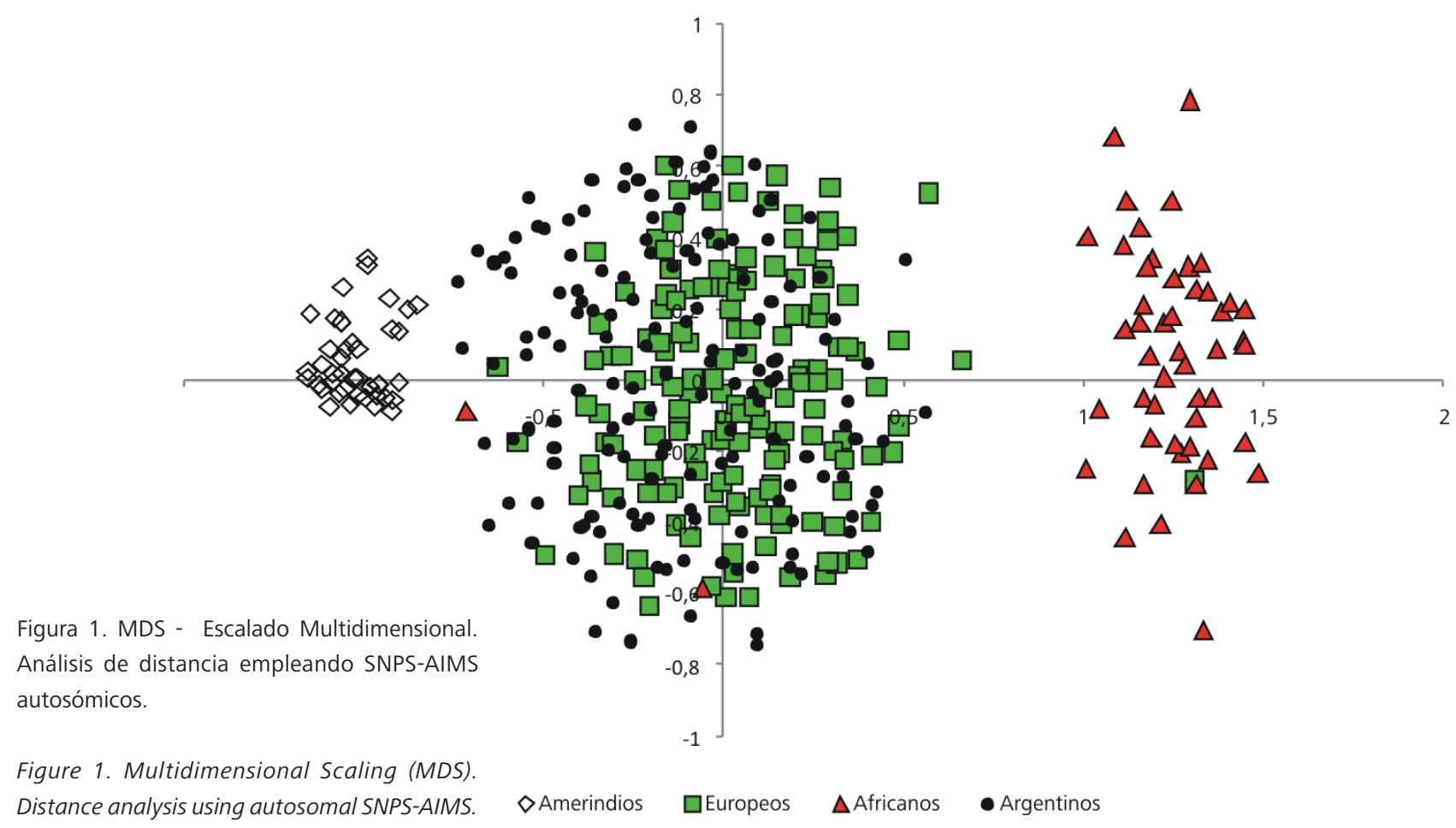

Tabla 1 donde puede observarse que más del $80 \%$ de las muestras mostró entre un 0 a $10 \%$ de componente amerindio, mientras que en casi el $98 \%$ pudo evidenciarse un componente europeo mayor al 50\%. Con respecto al componente africano, en casi la totalidad de las muestras $(98,6 \%)$ fue menor al $10 \%$.

\section{ADN Mitocondrial}

\section{Inserciones - Deleciones}

Sin tener en cuenta las inserciones frecuentes observadas en los tractos de polic se han detectado dos muestras con inserciones en posiciones poco habituales: 46.1T y 71.1G. La aparición de estas inserciones podría tener relación con la escasez de datos de secuencias mitocondriales para poblaciones amerindias ya que ambas fueron observadas en muestras tipificadas como pertenecientes a haplogrupos (hgs) amerindios. La muestra ABS236 presentó la deleción de seis pares de bases descripta para individuos de la población Chibcha - 106-111Del - (Santos y Barrantes 1994, Merriwether et al. 1995). El análisis de deleciones se llevó a cabo sin tener en cuenta las características en las posiciones 249, 290, 291, 298 y 524.

\section{Sustituciones - Transversiones}

El análisis de las regiones hipervariables I, II y III (HVR I, II y III) permitió caracterizar las sustituciones más frecuentes para la región HVR-I: 16189C (35 veces), 16362 C (37 veces), 16223T (60 veces) y 16519C (88 veces); mientras que para las regiones HVR-II y III las más frecuentes se presentan en las posiciones 73A y $263 \mathrm{G}$ (115 y 153 veces respectivamente). El mayor porcentaje de transversiones se detectó en las posiciones 16182 y 16183, observándose un cambio de $A$ por $C$ en ambos casos. Esto se condice con el aumento en la probabilidad de ocurrencia de transversiones en las zonas flanqueantes a los tractos de polic.

\section{Heteroplasmías}

La coexistencia, en una misma posición, de dos bases para una misma secuencia pudo detectarse en 8 muestras, en todos los casos en forma única. Las posiciones $\mathrm{y}$ los cambios observados fueron: 146Y, 153R, 16142Y, $16163 R, 16263 Y, 16318 R, 16327 Y$ y $16356 Y$

\section{Asignación de Haplogrupos (Hgs)}

Cada haplogrupo fue definido teniendo en cuenta el

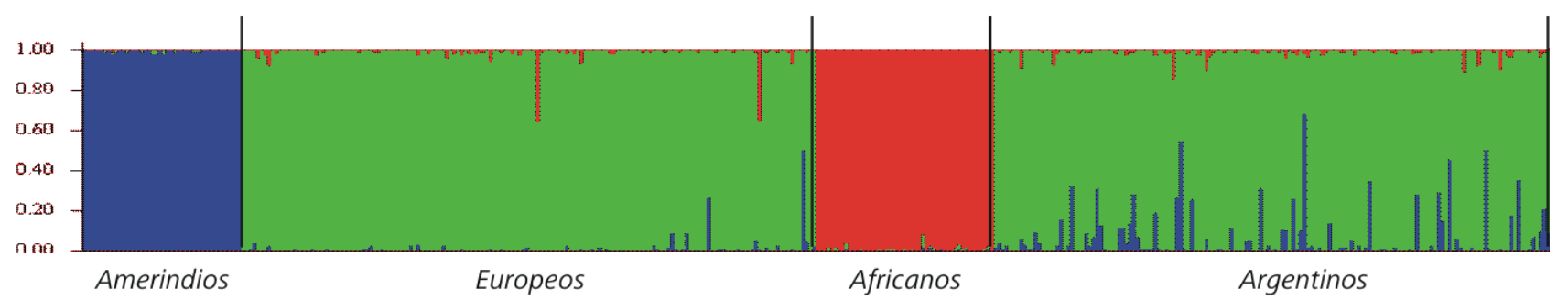

Figura 2. Gráfico de barras - STRUCTURE. Análisis de la composición étnica a nivel individual. 


\begin{tabular}{lccc}
\hline & Componente Amerindio & Componente Europeo & Componente Africano \\
\hline entre $\mathbf{0}$ y $\mathbf{1 0} \%$ & $81,69 \%$ de las muestras & $0 \%$ de las muestras & $98,6 \%$ de las muestras \\
entre $\mathbf{1 0}$ y $\mathbf{5 0} \%$ & $16,33 \%$ de las muestras & $1,96 \%$ de las muestras & $1,3 \%$ de las muestras \\
más de $\mathbf{5 0} \%$ & $1,96 \%$ de las muestras & $97,38 \%$ de las muestras & $0 \%$ de las muestras \\
\hline
\end{tabular}

Tabla 1. Proporción de Componentes Amerindios, Europeos y Africanos.

Table 1. Amerindian, European and African component proportions.

análisis de la región de control completa (HVR I, II y III) empleando la nomenclatura de Bandelt y Parson (2008). La presencia de mutaciones poco frecuentes y el análisis de SNPS en la región codificante permitieron definir subhaplogrupos derivados, algunos de los cuales no han sido descriptos previamente.

En el total de muestras analizadas se observaron los cuatro hgs amerindios: A2, B2, C1 y D (D1 y D4h3a), los cuales corresponden al $46 \%$ de la población total; en el 1,3\% de los individuos pudo establecerse el origen africano (L); 1,3\% fueron tipificados como pertenecientes al hg N1b (Europa y Oriente Medio) y los restantes $(51,14 \%)$ correspondieron a los hgs europeos más frecuentes (v.g. H, I, J, K, T, U, V, R y W ) En la Figura 3 se presenta la frecuencia de los diversos hgs detectados en la muestra de Buenos Aires analizada.

La frecuencia de hgs europeos fue mayor respecto a los hgs amerindios, observándose una diferencia del 5\%. Dentro de los amerindios, los hgs A2, B2 y C1 se encontraron en igual frecuencia (19\%) y en último lugar el Hg D con un 11,7\%. Para confirmar el estado de B2, que corresponde al $\mathrm{Hg} B$ amerindio, se analizó el SNP en la posición 3547 de región codificante; para detectar los subhaplogrupos de C1b y C1c se analizó la posición 1888, mientras que para C1d la posición 7697. Dentro del Hg D el 14\% correspondió al subhaplogrupo D4h3 mientras que el resto de las muestras fueron tipificadas como D1. En un trabajo posterior tres de las muestras identificadas como D1 fueron sub-clasificadas como D1g2, D1h1 y D1h1a1 (Bodner et al. 2012)

El hg Europeo más frecuente fue el $\mathrm{H}$ con 22,7\% observándose una gran diversidad de hgs europeos (I, J, K, T, U, V, R y W). Los subhaplogrupos J1c y K2

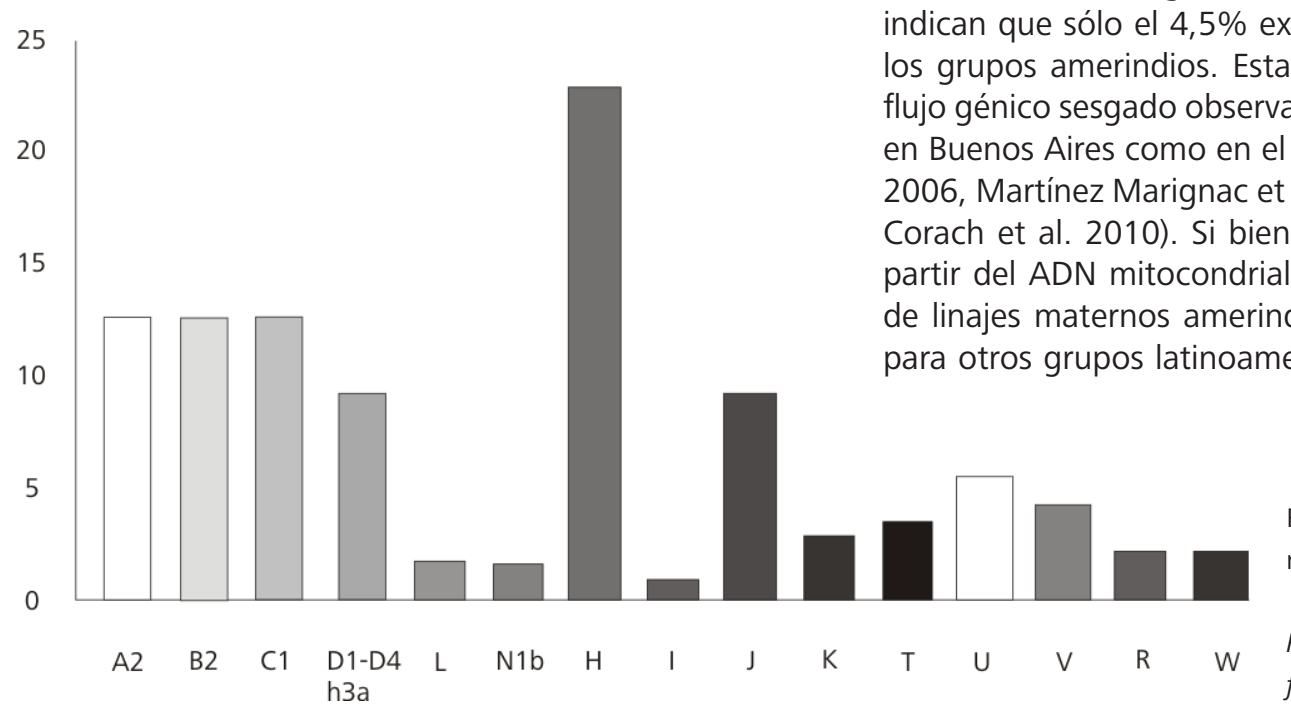

fueron confirmados con los SNP 14798 y 9716 de región codificante respectivamente. Para el hg africano $L$, que representó el 1,3\%, pudieron identificarse los subhaplogrupos LOa2 y L2C.

La distribución de las frecuencias de subhaplogrupos amerindios y europeos se presenta en la tabla 2.

En la figura 4 se muestra el gráfico de redes "network" en el que se representan los haplotipos mitocondriales de la muestra analizada. Pueden observarse las dos muestras tipificadas como de origen africano muy alejadas del resto, y un agrupamiento de las muestras coincidente con los hgs a los que fueron asignadas.

\section{SNPS Cromosoma Y}

La investigación de los SNPs en la NRY, con la finalidad de asignar un origen geográfico en base a las variantes observadas en las poblaciones ancestrales, mostró un $95,5 \%$ de origen europeo ( $E, G, I, J, K, L, R 1 a$ y R1b1b2a) y un $4,5 \%$ de origen amerindio (Q) (Tabla 3).

Estos porcentajes fueron confirmados con los resultados del SNP M3-DYS199 donde se obtuvo la variante T para siete de las ciento cincuenta y cuatro muestras seleccionadas.

\section{Discusión y conclusiones}

Los marcadores de herencia uniparental (ADN mitocondrial y Y-SNP) evidenciaron resultados similares en cuanto a la proporción dispar de componente amerindio, concordante con otras poblaciones americanas analizadas. A diferencia del cuadro aportado por los linajes maternos, los resultados emergentes del análisis del cromosoma $Y$ indican que sólo el 4,5\% exhibe el Y-SNP asociado con los grupos amerindios. Estas observaciones ratifican el flujo génico sesgado observado en otros trabajos, ya sea en Buenos Aires como en el resto del país (Corach et al. 2006, Martínez Marignac et al. 2004, Avena et al. 2006, Corach et al. 2010). Si bien los resultados obtenidos a partir del ADN mitocondrial mostraron una proporción de linajes maternos amerindios menor a los obtenidos para otros grupos latinoamericanos (Batista dos Santos

Figura 3. Porcentaje de haplogrupos mitocondriales.

Figure 3. Mitochondrial haplogroup frequency. 


\begin{tabular}{cccccccccccc}
\hline SubHg & $\%$ & SubHg & $\%$ & SubHg & $\%$ & SubHg & $\%$ & SubHg & $\%$ & SubHg & $\%$ \\
\hline A2 & 9.09 & C1b6 & 1.30 & H3al1a & 0.65 & J1c1 & 0.65 & T2b & 1.30 & V & 3.90 \\
A2I & 1.30 & C1C & 1.95 & H5 & 1.30 & J1d & 0.65 & U2d & 0.65 & W & 1.95 \\
A2m & 1.95 & C1d & 3.25 & H6 & 0.65 & J2 & 0.65 & U4 & 0.65 & & \\
B2 & 5.84 & D1 & 3.25 & HV0 & 1.30 & J2a & 1.30 & U4a2 & 0.65 & & \\
B2b & 1.95 & D1e & 0.65 & I2 & 0.65 & K1a & 1.30 & U4a2a & 0.65 & & \\
B2C & 1.30 & D1f & 3.25 & J1b & 0.65 & R0 & 1.30 & U5a & 0.65 & & \\
B4 & 0.65 & D4h3a & 1.95 & J1b1 & 0.65 & R0a & 0.65 & U5a1 & 0.65 & & \\
B4b & 2.60 & H & 15.58 & J1b2 & 0.65 & T1a & 1.30 & U5b & 0.65 & & \\
C1b & 5.84 & H1a & 1.95 & J1c & 3.90 & T2 & 0.65 & U6b & 0.65 & & \\
\hline
\end{tabular}

Tabla 2. Frecuencias de Subhaplogrupos mitocondriales.

Table 2. Mitochondrial subHaplogroups frequencies.

et al. 1999, Bertoni et al. 2005, Campos-Sánchez et al. 2006), el porcentaje de los mismos fue superior al $45 \%$.

El alto porcentaje de linajes maternos pre-hispánicos se debe principalmente a los sucesos de poblamiento ocurridos en el actual territorio de Argentina, donde la principal expansión de la población tuvo lugar con la llegada masiva de hombres europeos. La escasa llegada de mujeres provenientes de Europa durante el inicio de la conquista y el prolongado contacto de los conquistadores con mujeres nativas trajo como consecuencia el elevado nivel de desbalance respecto de los linajes maternos y paternos con mayoría nativa en los maternos.
El primer contacto interétnico produjo una reducción severa de la población nativa masculina como consecuencia de las sucesivas guerras ocurridas con la finalidad de ocupar el territorio. Así mismo, como consecuencia de una decisión política de poblamiento del territorio conquistado, sumado a la falta de población femenina de origen europeo, el mestizaje entre hombres españoles y mujeres nativas fue un evento muy frecuente que dio origen a descendientes con linajes paternos europeos. La notable disminución de los hombres nativos, y el flujo génico sesgado debido a que los nativos no tenían descendencia con mujeres europeas, fueron dos factores fundamentales que determinaron la frecuencia de linajes paternos. Otro factor que contribuye a la proporción

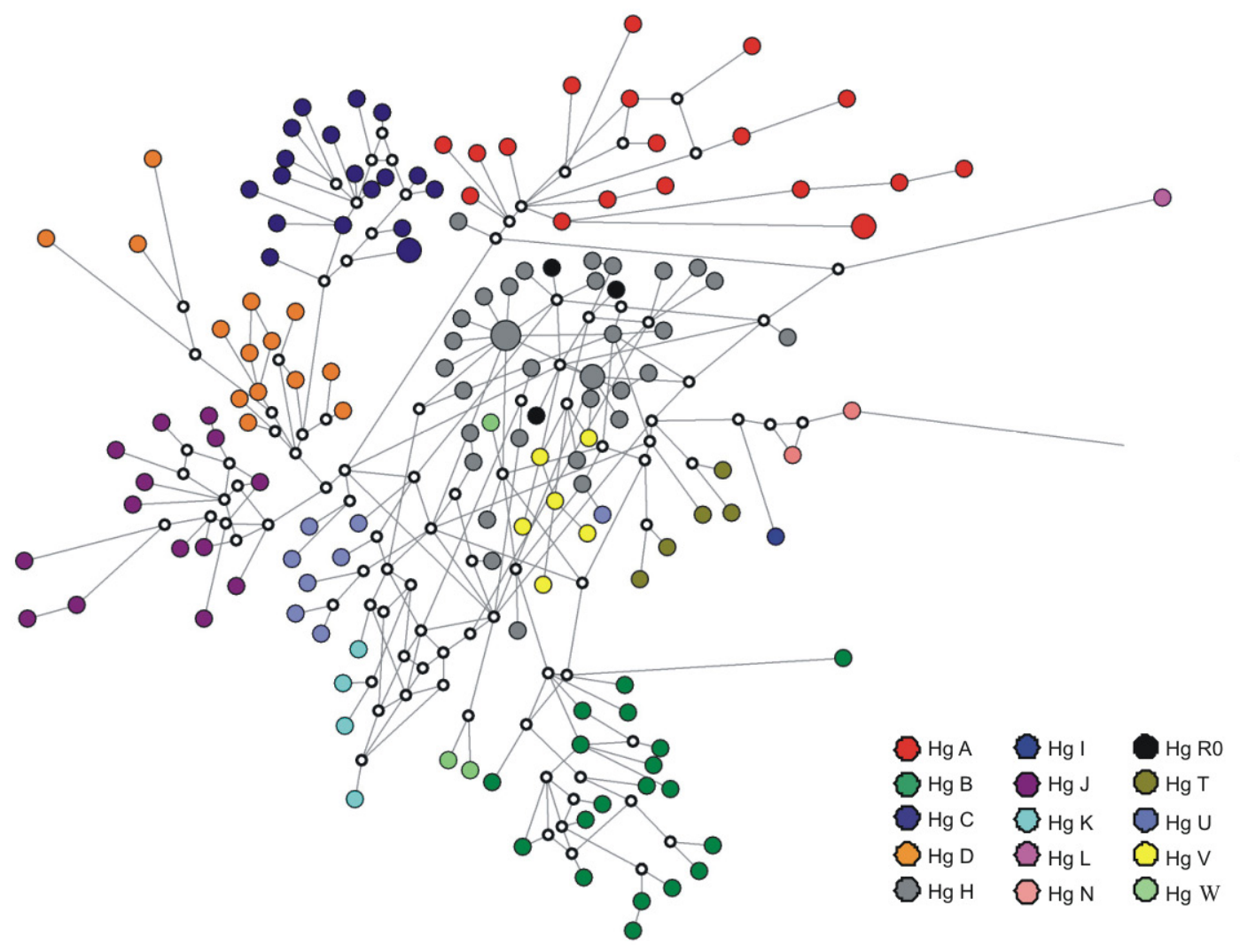

Figura 4. Gráfico de Network de los haplotipos mitocondriales analizados.

Figure 4. Network graph of mitochondrial haplotypes analyzed. 


\begin{tabular}{cccc}
\hline $\mathbf{H q}$ & $\mathbf{\%}$ & $\mathbf{H q}$ & $\mathbf{\%}$ \\
\hline $\mathbf{E}$ & 9.804 & $\mathbf{K}$ & 1.961 \\
$\mathbf{G}$ & 5.882 & $\mathbf{L}$ & 0.654 \\
$\mathbf{I}$ & 9.15 & $\mathbf{Q}$ & 4.575 \\
$\mathbf{J}$ & 16.99 & $\mathbf{R 1 a}$ & 2.614 \\
& & $\mathbf{R} 1 \mathbf{b} \mathbf{1} \mathbf{b} \mathbf{2 a}$ & 48.37 \\
\hline
\end{tabular}

Tabla 3. Frecuencia de haplogrupos de cromosoma Y.

Table 3. Chromosome Y haplogroup frequency.

observada es la inmigración masiva proveniente principalmente de Italia y España (1850 -1930) como consecuencia de la situación social existente en los países europeos como así también por la política inmigracionista existente en Argentina. Estas migraciones habrían tenido un fuerte impacto en la población de Buenos Aires ya que debido a su mayor industrialización habría atraído a los recién llegados.

Si bien entre los años 1580 y 1810 se produjo una entrada masiva de esclavos africanos, la escasa población de origen Africano evidenciada tanto por el bajo porcentaje de linajes maternos como paternos podría deberse por un lado a barreras sociales existentes en aquel momento que determinaron un bajo grado de mestizaje, como también a la alta tasa de mortandad sufrida por este grupo como consecuencia de las guerras de la independencia y enfermedades. Los resultados obtenidos en este trabajo, si bien son concordantes con otros previos para la población Argentina, muestran un componente africano muy bajo comparado con otros países de América como Estados Unidos, Uruguay y Brasil, donde el grado de mezcla étnica con la población africana fue superior (Bravi et al. 1997, Gonçalves et al. 2007).

Mediante el uso de marcadores de herencia biparental (SNPs-AIMs) pudo evidenciarse un componente europeo mayoritario. A pesar de esta tendencia general en la composición génica, la variación interindividual mostró en todos los casos un alto grado de heterogeneidad, con diferente proporción de los tres componentes ancestrales. Aún en casos de linajes paternos y maternos amerindios se observó esta tendencia, reflejando la dinámica de la historia demográfica reciente de Argentina, donde los individuos con una proporción mayor de ancestro europeo estarían relacionados con inmigraciones recientes mientras que aquellos en donde se observó un grado de mezcla con mayor proporción amerindia podrían ser el resultado de la descendencia entre las poblaciones originarias y los primeros conquistadores que habían arribado al continente 500 años atrás.

La mayor contribución africana detectada con los SNPsAIMs, comparada con los marcadores uniparentales, se relacionaría con los procesos iniciales de mestizaje donde este grupo habría tenido un rol significativo quedando representado en estos segmentos recombinantes, mientras que los linajes uniparentales habrían desaparecido.

Con los anteriormente mencionado cabe señalar que, si bien el espacio muestral analizado representa solo una pequeña fracción de la población total de la provincia de Buenos Aires, lo que podría relacionarse con una subestructura poblacional, los resultados son concordantes con los observado para el resto del país y otros países de Latinoamérica. Este desplazamiento del acervo génico pre-hispánico observado se evidenció principalmente en los linajes paternos, siendo reemplazados en su mayoría por aportes europeos. En relación a los linajes mitocondriales, si bien se han preservado en mayor proporción, también han sido desplazados en más del 50\%, por linajes europeos.

Buenos Aires, 13 de Abril de 2012

\section{Agradecimientos}

Este trabajo fue realizado, en parte, con financiación de los subsidios UBACyT W744 (2011-2014) y PIP 112200801-02836 (2009-2011) CONICET otorgados a DC y con fondos genuinos del SHDG. CB y DC son miembros de la CIC CONICET.

\section{Bibliografia}

Andrews, G. R. 1980. The Afro-Argentines of Buenos Aires, 1800/1900. Wisconsin: University of Wisconsin Press, 286 pp.

Andrews, R.M., I. Kubacka, P.F. Chinnery, R.N. Lightowkers, D.M. Turnbull y N. Howell. 1999. Reanalysis and revision of the Cambridge reference sequence for human mitochondrial DNA. Nature Genetics 23: 147

Avena, S.A., A.S. Goicoechea, J. Dugoujon, M. Slepoy, A. Slepoy y F.R. Carnese. 2001. Análisis Antropogenético de los Aportes Indígena y Africano en Muestras Hospitalarias de la Ciudad de Buenos Aires. Revista Argentina de Antropología, Biológica 3 (1):79-99.

Avena, S.A., A.S. Goicoechea, J. Rey, J.M. Dugoujon, C.B. Dejean y F.R. Carnese. 2006. Mezcla genica en una muestra poblacional de la ciudad de Buenos Aires. Medicina (Buenos Aires) 66: 113-118.

Bandelt, H.J. y W. Parson. 2008. Consistent treatment of length variants in the human mtDNA control region: a reappraisal. International Journal of Legal Medicine 122: 11-21.

Batista dos Santos, S.E., J.D. Rodrigues, A.K. Ribeiro-dosSantos y M.A. Zago. 1999. Differential contribution of indigenous men and women to the formation of an urban population in the Amazon region as revealed by mtDNA and Y-DNA. American Journal of Physical Anthropology 109 (2):175-180

Bertoni, B., L. Jin, R. Chakraborty y M. Sans. 2005. Directional mating and a rapid male population expansion in a hybrid Uruguayan population. American Journal of Human Biology 17 (6):801-808 
Bodner, M., A.U. Perego, G. Huber, L. Fendt, A.W. Röck, B. Zimmermann, A. Olivieri, A. Gomez-Carballa, H. Lancioni, N. Angerhofer, M.C. Bobillo, D. Corach, S.R. Woodward, A. Salas, A. Achilli, A. Torroni, H. Bandelt y W. Parson. 2012. Rapid coastal spread of First Americans: Novel insights from South America's Southern Cone mitochondrial genomes. Genome Research 22 (5): 811820.

Brandstätter, A., A. Salas, H. Niederstätter, C. Gassner, A. Carracedo y W. Parson. 2006. Dissection of mitocondrial suprhaplogroup $\mathrm{H}$ using coding region SNPs. Electrophoresis 27(13): 2541-2550.

Bravi, C.M., M. Sans, G. Bailliet, V.L. Martínez-Marignac, M. Portas, I. Barreto, C. Bonilla y N.O Bianchi. 1997. Characterization of mitochondrial DNA and Y-chromosome haplotypes in a Uruguayan population of African ancestry. Human Biology 69 (5): 641-652.

Campos-Sánchez, R., R. Barrantes, S. Silva, M. Escamilla, A. Ontiveros, H. Nicolini, R. Mendoza, R. Muñoz y H. Raventos. 2006. Genetic structure analysis of three Hispanic populations from Costa Rica, Mexico, and the southwestern United States using Y-chromosome STR markers and mtDNA sequences. Human Biology 78 (5): 551-563

Cann, R.L., M. Stoneking y A.C. Wilson. 1987. Mitochondrial DNA and human evolution. Nature 325: 31-36.

Corach, D., M. Marino y A. Sala. 2006. Relevant Genetic Contribution of Amerindian to the extant population of Argentina. International Congress Series 1288: 397-399.

Corach, D., O. Lao, C. Bobillo, K. van Der Gaag, S. Zuniga, M. Vermeulen, K. van Duijn, M. Goedbloed, P.M. Vallone, W. Parson, P. de Knijff y M. Kayser. 2010. Inferring continental ancestry of argentineans from Autosomal, Y-chromosomal and mitochondrial DNA. Annals of Human Genetics 74 (1): 65-76.

De Knijff, P. 2000. Messages through bottlenecks: On the Combined Use of Slow and Fast Evolving Polymorphic Markers on the Human Y Chromosome. American Journal of Human Genetics 67: 1055-1061.

Gonçalves, V.F., C.M. Carvalho, M.C. Bortolini, S.P. Bydlowski y S.D. Pena. 2007. The phylogeography of African Brazilians. Hum Heredety 65 (1): 23-32.

INDEC (Instituto Nacional de Estadística y Censo), www. indec.gov.ar

Kersbergen, P., K.V. Duijn, A.D. Kloosterman AD, J.T. Dunnen, M. Kayser y P. de Kniff. 2009. Developing a set of ancestry-sensitive DNA markers reflecting continental origins of humans. BMC Genetics 10: 69.

La Ciudad en el Censo Nacional de 1914. Tercer Censo General. Población de Buenos Aires [en línea] 2008, vol. 5 ISSN 1668- 5458 http://redalyc.uaemex.mx/src/inicio/ ArtPdfRed.jsp?iCve=74011761007.

Mandrini, R. 2005. Los pueblos originarios de la Argentina. La visión del otro. Buenos Aires: Editorial Universitaria de Buenos Aires EUDEBA.

Mandrini, R. 2008. La Argentina Aborígen. De los primeros pobladores a 1910. Buenos Aires: Siglo XXI Editores Argentina.

Martínez Marignac, V. L., B. Bertoni, E.J. Parra y N.O. Bianchi. 2004. Characterization of admixture in an urban sample from Buenos Aires, Argentina, using uniparentally and biparentally inherited genetic markers. Human Biology 76: 543-557.

Martínez Sarasola, C. 2011. Nuestros paisanos los indios. Buenos Aires: Editorial del Nuevo Extremo S.A. Primera edición.

Merriwether, D.A., R.E. Ferrell y F. Rothhammer. 1995. mtDNA D-loop 6-bp deletion found in the Chilean Aymara: not a unique marker for Chibcha-speaking Amerindians. American Journal of Human Genetics 56: 812-813.

Pritchard, J. K., M. Stephens, y P. Donnelly. 2000. Inference of population structure using multilocus genotype data. Genetics 155: 945-959.

Rowley, J.A. 2005. The Transatlantic Slave Trade. University of Nebraska Press, Lincoln and London.

Santos, M., R. Barrantes. 1994. D-loop mtDNA deletion as a unique marker of Chibchan Amerindians. American Journal of Human Genetics 55: 413-414.

Underhill, P.A. y T. Kivisild. 2007. Use of Y Chromosome and Mitochondrial DNA Population Structure in Tracing Human Migrations. Annual Review of Genetics 41: 539564.

Vigilant, L., M. Stoneking, H. Harpending, K. Hawkes y A.C. Wilson. 1991. African Populations and the Evolution of Human Mitochondrial DNA. Science, New Series 253 (5027): 1503-1507.

Zuccarelli, G., E. Alechine, M. Caputo, C. Bobillo, D. Corach, A. Sala. 2011. Rapidscreening for Native American mitochondrial and Y-chromosome haplogroups detection in routine DNA analysis. Forensic Science International: Genetics 5 (2): 105-108. 